\title{
Influence of Drip-irrigation, Manure and Fertilizers on Production of Planting Material in Sisal (Agave sisalana Perr. Ex Engelm.)
}

\author{
Sitangshu Sarkar*, A.K. Jha, Bijan Majumdar and A.R. Saha \\ ICAR-Central Research Institute for Jute and Allied Fibres, Nilgunj, Barrackpore, \\ Kolkata-700120, India \\ *Corresponding author
}

\section{A B S T R A C T}

\section{Keywords}

Sisal, Agave sisalana, Sucker production, Dripirrigation, Manure, Fertilizer

Article Info

Accepted:

12 August 2018 Available Online:

10 September 2018
Field experiment was conducted during 2006-07 to 2017-18 (12 years) at Sisal Research Station $\left(22.041^{\circ} \mathrm{N}, 84.295^{\circ} \mathrm{E}, 267 \mathrm{~m} \mathrm{AMSL}\right)$, Bamra, Odisha, India to study the effect of manure (FYM), drip-irrigation and fertilizers on the number of sucker production in sisal (Agave sisalana Perr. Ex Engelm.). It was found that application of manure (FYM @ 5 t/ha), drip-irrigation (with discharge rate of $4 \mathrm{l} / \mathrm{hr}$ for $2 \mathrm{hrs}$ at 2 weeks interval during $1^{\text {st }}$ April-15 ${ }^{\text {th }}$ June), and fertilizers $\left(\mathrm{N}_{60} \mathrm{P}_{30} \mathrm{~K}_{60}\right.$ or $\left.\mathrm{N}_{120} \mathrm{P}_{30} \mathrm{~K}_{60} \mathrm{~kg} / \mathrm{ha}\right)$ produced a total of $142.6 \mathrm{x}$ $10^{3}$ suckers/ha during $7^{\text {th }}$ to $12^{\text {th }}$ crop year. Production of number of suckers diminishes gradually from $7^{\text {th }}$ year to $12^{\text {th }}$ year, after which the sisal crop completes its life cycle by production of flowering stalk and eventual natural dying. The produced $142.6 \times 10^{3}$ number of suckers is sufficient for covering 28.5 ha new sisal plantation. The quantum of suckers so produced adds farmers' income by ₹ 5.7 lakh at present price rate of sisal planting material ( $₹ 4,000$ for 1000 suckers).

\section{Introduction}

Sisal (Agave sp), a xerophytic member of Aaparagaceae family, from which commercial hard fibre is produced from its leaf. Number of species of Agaves namely A. sisalana, A. cantala, A. vera-cruz, A. amaniensis, A. angustifolia, A. fourcryodes and others are cultivated (Sarkar and Jha, 2017). However, among the different species, A. sisalana contributes nearly $85 \%$ of the total sisal fibre production of the World. In India sisal is cultivated in western Odisha, Jharkhand, Chhattisgarh, and in scattered areas of Maharashtra, Andhra Pradesh and Karnataka (Sarkar, 2015). In Indian condition seed is not produced in sisal might be due to lack of pollinating bat species and therefore, the crop is propagated through vegetative means namely bulbils and suckers. Bulbils are small plantlets with 2-3 small leaves produced on the flowering stalk of mature sisal plant once in 10-12 years or so. Bulbils are so tiny that it require extra care in nurseries for about a year and then it can be used as planting material in the main field of sisal. Whereas, suckers are bigger sized plantlets started producing from the roots of sisal plant after years of active growth and continues up to the end of life span of sisal (say 12 years). Suckers are generally stout enough and are commonly used as propagating material (Sarkar et al., 
2010). Earlier it was recorded that planting through bulbils caused $9 \%$ or more mortality, whereas, planting through suckers caused minimum mortality of the sisal plantlets (Reddy, 1966). Recent report quantified the number of suckers produced from a healthy sisal plantation for 2 consecutive years only (Sarkar et al., 2017). Virtually there is no information available for influence of manure (FYM), drip-irrigation, and fertilizer application on sucker production capability of sisal for the whole economic life span of sisal. So, it is necessary to generate information about number of sucker production for planting of new sisal plantation. Therefore, a field experiment was conducted to study the effect of manure (FYM), drip-irrigation, and fertilizers on production of number of suckers (planting material) in sisal (Agave sisalana Perr. Ex Engelm.).

\section{Materials and Methods}

A field experiment was conducted during 2006-07 to 2017-18 (12 years) at the Sisal Research Station (located at $22.041^{\circ} \mathrm{N}$, $84.295^{\circ} \mathrm{E}, 267 \mathrm{~m} \mathrm{AMSL}$ ) a regional research station of ICAR-CRIJAF), at Bamra, in Sambalpur district of Odisha, India to study the effect of drip-irrigation, manure and different doses of fertilizers on production of sucker (planting material) in sisal (Agave sisalana Perr. Ex Engelm.). The experiment soil was acidic in reaction $\mathrm{pH}(1: 2.5 \mathrm{w} / \mathrm{v})$ in water 5.20 , low in organic carbon $3.4 \mathrm{~g} / \mathrm{kg}$, having available nitrogen of $186 \mathrm{~kg} / \mathrm{ha}$, available phosphorus of $31.7 \mathrm{~kg} / \mathrm{ha}$ and available potassium of $116 \mathrm{~kg} / \mathrm{ha}$. The experiment was laid in 3 factor split-split plot design with 2 levels of manure $\left(\mathrm{M}_{1}=\right.$ no manure, $\mathrm{M}_{2}=\mathrm{FYM} @ 5 \mathrm{t} / \mathrm{ha}$ ) in main plot, 2 levels of irrigation $\left(I_{1}=\right.$ no irrigation; $I_{2}$ : drip irrigation with discharge rate of $4 \mathrm{l} / \mathrm{hr}$ for 2 hours at 2 weeks interval during $1^{\text {st }}$ April to $15^{\text {th }}$ June) in sub plot and 4 levels of fertilizers $\left[\mathrm{N}_{1}=\right.$ no fertilizer, $\mathrm{N}_{2}=\mathrm{N}_{0} \mathrm{P}_{30} \mathrm{~K}_{60}, \quad \mathrm{~N}_{3}=$
$\mathrm{N}_{60} \mathrm{P}_{30} \mathrm{~K}_{60}$, and $\left.\mathrm{N}_{4}=\mathrm{N}_{120} \mathrm{P}_{30} \mathrm{~K}_{60}\right]$ in sub-sub plots with individual plot size of $8 \mathrm{~m} \times 6 \mathrm{~m}$ replicated three times. Healthy suckers of sisal were planted in the recommended double row planting system $[(1 \mathrm{~m} \times 1 \mathrm{~m}) \times 3 \mathrm{~m}]$ in the month of July, 2006. Other standard recommended agronomic practices were followed to raise the experimental sisal plantation. The suckers produced in the plots were collected for 6 consecutive years starting from 2012-13 and up to 2017-18. So, after 12 years of crop age, the sisal plantation produced flower stalks and started dying which is a common phenomenon in sisal. The 6 years data on numbers of sucker production were processed and analysed by statistical software IBM SPSS Statistics v. 24.

\section{Results and Discussion}

\section{Effect of manure on numbers of sucker production in sisal}

Application of manure (FYM @ 5 t/ha) showed beneficial effect on number of suckers production in sisal (Table 1). In the first year of sucker collection (2012-13), $22.93 \times 10^{3}$ suckers were collected, which reduced gradually in the subsequent years $\left(22.27 \times 10^{3}\right.$ in 2013-14; $15.21 \times 10^{3}$ in 2014-15 and so on). During this 6 years sucker collection period, a total of $80.65 \times 10^{3}$ suckers were produced from the manure applied plots. Application of manure increased sucker production by $52.7 \%$ as compared to no manure application.

\section{Effect of drip irrigation on numbers of sucker production}

Drip irrigation (with discharge rate of $4 \mathrm{l} / \mathrm{hr}$ for 2 hours at 2 weeks interval during $1^{\text {st }}$ April to $15^{\text {th }}$ June) in sisal had significant effect on number of sucker production of sisal (Table 2). Production of suckers decreased over years in both irrigated $\left(20.02 \times 10^{3}\right.$ in $2012-13$ to $2.61 \times 10^{3}$ in 2017-18) and unirrigated 
condition $\left(15.55 \times 10^{3}\right.$ in $2012-13$ to $2.33 \mathrm{x}$ $10^{3}$ in 2017-18). Application of drip irrigation in sisal produced $32.8 \%$ more number of suckers as compared to no irrigation.

\section{Effect of nutrients on numbers of sucker production}

Production of suckers varied non-significantly with different doses of nutrients, except in 2014-15, when $\mathrm{N}_{60} \mathrm{P}_{30} \mathrm{~K}_{60}$ produced the highest number of suckers $\left(23.33 \times 10^{3}\right)$. Over a period of 6 years of sucker collection, the highest number of total suckers $\left(103.98 \times 10^{3}\right)$ were recorded with $\mathrm{N}_{120} \mathrm{P}_{30} \mathrm{~K}_{60}$ (Table 3).

Interaction effect of manure and dripirrigation on numbers of sucker production

Application of manure and drip irrigation showed positive interaction effect on number of sucker production in sisal (Table 4). In the first year of sucker collection (2012-13), the highest number of suckers produced was
$25.33 \times 10^{3}$, which was about $72.2 \%$ more as compared to no manure and no irrigation $\left(10.57 \times 10^{3}\right)$. In the 6 years period of observation, the number of sucker produced with manure and irrigation was $88.86 \times 10^{3}$, which was $40.1 \%$ more as compared to the no manure and no irrigation treatment.

\section{Interaction effect of manure and nutrients on numbers of sucker production}

Application of manure and nutrient in sisal showed significant positive effect on number of sucker production in sisal (Table 5). Application of nutrients $\left(\mathrm{N}_{60} \mathrm{P}_{30} \mathrm{~K}_{60}\right.$ and $\mathrm{N}_{120} \mathrm{P}_{30} \mathrm{~K}_{60}$ ) along with manure (FYM @ 5 t/ha) produced $34.9 \times 10^{3}$ and $36.0 \times 10^{3}$ suckers in 2012-13. In the subsequent years, the $\mathrm{N}_{60} \mathrm{P}_{30} \mathrm{~K}_{60}$ and $\mathrm{N}_{120} \mathrm{P}_{30} \mathrm{~K}_{60}$ levels of nutrients along with manure produced the maximum number of suckers. Over the 6 years period, $\mathrm{N}_{120} \mathrm{P}_{30} \mathrm{~K}_{60}$ level of nutrient and manuring (FYM @ 5 t/ha) produced the highest number of suckers $\left(126.51 \times 10^{3}\right)$.

Table.1 Main effect of manure (M) on number of suckers production/ha ('000) in sisal

\begin{tabular}{|l|c|c|c|c|c|c|c|}
\hline Manure levels & \multicolumn{7}{|c|}{ Number of suckers produced/ha ('000) } \\
\cline { 2 - 10 } & $2012-13$ & $2013-14$ & $2014-15$ & $2015-16$ & $2016-17$ & $2017-18$ & Total \\
\hline No manure & 12.64 & 14.28 & 11.57 & 6.45 & 5.62 & 2.24 & 52.80 \\
\hline Manure (5t/ha) & 22.93 & 22.27 & 15.21 & 11.19 & 6.33 & 2.70 & 80.65 \\
\hline SEm士 & 0.491 & 0.820 & 0.367 & 0.445 & 0.132 & 0.065 & 1.158 \\
\hline LSD (0.05) & 1.700 & 2.839 & 1.269 & 1.541 & 0.458 & 0.224 & 4.008 \\
\hline
\end{tabular}

Table.2 Main effect of irrigation (I) on number of suckers production/ha ('000) in sisal

\begin{tabular}{|l|c|c|c|c|c|c|c|}
\hline Irrigation levels & \multicolumn{7}{|c|}{ Number of suckers produced/ha ('000) } \\
\hline & $2012-13$ & $2013-14$ & $2014-15$ & $2015-16$ & $2016-17$ & $2017-18$ & Total \\
\hline No irrigation & 15.55 & 15.74 & 11.34 & 7.69 & 4.65 & 2.33 & 57.31 \\
\hline Drip irrigation & 20.02 & 20.81 & 15.44 & 9.95 & 7.30 & 2.61 & 76.14 \\
\hline SEm \pm & 0.491 & 0.820 & 0.367 & 0.445 & 0.132 & 0.065 & 1.158 \\
\hline LSD (0.05) & 1.700 & 2.839 & 1.269 & 1.541 & 0.458 & 0.224 & 4.008 \\
\hline
\end{tabular}


Table.3 Main effect of nutrients (N) on number of suckers production/ha ('000) in sisal

\begin{tabular}{|l|c|c|c|c|c|c|c|}
\hline Nutrient levels & \multicolumn{7}{|c|}{ Number of suckers produced/ha ('000) } \\
& $2012-13$ & $2013-14$ & $2014-15$ & $2015-16$ & $2016-17$ & $2017-18$ & Total \\
\hline $\mathbf{N}_{0} \mathbf{P}_{0} \mathbf{K}_{0}$ & 6.33 & 6.59 & 5.55 & 4.06 & 3.63 & 1.98 & 28.14 \\
\hline $\mathbf{N}_{0} \mathbf{P}_{30} \mathbf{K}_{60}$ & 10.65 & 12.02 & 7.17 & 5.67 & 4.41 & 2.21 & 42.14 \\
\hline $\mathbf{N}_{60} \mathbf{P}_{30} \mathbf{K}_{60}$ & 26.10 & 21.08 & 23.33 & 11.70 & 7.31 & 3.12 & 92.65 \\
\hline $\mathbf{N}_{120} \mathbf{P}_{30} \mathbf{K}_{60}$ & 28.06 & 33.43 & 17.51 & 13.86 & 8.55 & 2.57 & 103.98 \\
\hline SEm \pm & 0.795 & 1.459 & 0.529 & 1.008 & 0.469 & 0.173 & 1.829 \\
\hline LSD $(\mathbf{0 . 0 5})$ & NS & NS & 1.545 & NS & NS & NS & NS \\
\hline
\end{tabular}

Table.4 Interaction effect of manure (M) and irrigation (I) on number of Sucker production in sisal

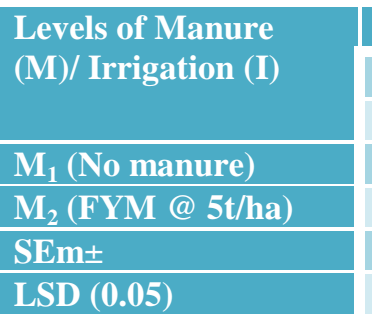

\begin{tabular}{|c|c|c|c|c|c|c|c|c|c|c|c|c|c|}
\hline \multicolumn{14}{|c|}{ Number of suckers produced per ha ('000) } \\
\hline \multicolumn{2}{|c|}{ 2012-13 } & \multicolumn{2}{|c|}{ 2013-14 } & \multicolumn{2}{|c|}{ 2014-15 } & \multicolumn{2}{|c|}{ 2015-16 } & \multicolumn{2}{|c|}{ 2016-17 } & \multicolumn{2}{|c|}{ 2017-18 } & \multicolumn{2}{|c|}{ Total } \\
\hline $\mathrm{I}_{1}$ & $\mathrm{I}_{2}$ & $\mathrm{I}_{1}$ & $\mathrm{I}_{2}$ & $\mathrm{I}_{1}$ & $\mathrm{I}_{2}$ & $\mathrm{I}_{1}$ & $\mathrm{I}_{2}$ & $\mathrm{I}_{1}$ & $\mathrm{I}_{2}$ & $\mathrm{I}_{1}$ & $\mathrm{I}_{2}$ & $\mathrm{I}_{1}$ & $\mathrm{I}_{2}$ \\
\hline 10.57 & 14.71 & 12.18 & 16.39 & 7.51 & 15.63 & 5.39 & 7.51 & 4.35 & 6.88 & 2.20 & 2.29 & 42.19 & 63.41 \\
\hline 20.53 & 25.33 & 19.31 & 25.23 & 15.18 & 15.25 & 10.00 & 12.39 & 4.94 & 7.72 & 2.47 & 2.92 & 72.43 & 88.86 \\
\hline \multicolumn{2}{|c|}{0.695} & \multicolumn{2}{|c|}{1.375} & \multicolumn{2}{|c|}{0.519} & \multicolumn{2}{|c|}{0.630} & \multicolumn{2}{|c|}{0.187} & \multicolumn{2}{|c|}{0.092} & \multicolumn{2}{|r|}{38} \\
\hline \multicolumn{2}{|c|}{2.404} & \multicolumn{2}{|c|}{4.015} & \multicolumn{2}{|c|}{1.795} & \multicolumn{2}{|c|}{2.179} & \multicolumn{2}{|c|}{0.647} & \multicolumn{2}{|c|}{0.317} & \multicolumn{2}{|c|}{5.669} \\
\hline
\end{tabular}

Table.5 Interaction effect of manure $(\mathrm{M})$ and nutrients $(\mathrm{N})$ on number of suckers production/ha ('000) in sisal

\begin{tabular}{|c|c|c|c|c|c|}
\hline \multirow[t]{2}{*}{ Year } & \multirow[t]{2}{*}{ Treatments } & \multicolumn{4}{|c|}{ Number of suckers produced/ha ('000) } \\
\hline & & $\mathrm{N}_{0} \mathrm{P}_{0} \mathrm{~K}_{0}$ & $\mathrm{~N}_{0} \mathrm{P}_{30} \mathrm{~K}_{60}$ & $\mathrm{~N}_{60} \mathrm{P}_{30} \mathrm{~K}_{60}$ & $\mathrm{~N}_{120} \mathrm{P}_{30} \mathrm{~K}_{60}$ \\
\hline \multirow[t]{4}{*}{ 2012-13 } & $\mathrm{M}_{1}$ (No manure) & 5.61 & 7.53 & 17.29 & 20.12 \\
\hline & 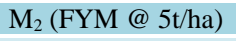 & 7.06 & 13.76 & 34.90 & 36.00 \\
\hline & $\mathrm{SEm} \pm$ & \multicolumn{4}{|c|}{0.562} \\
\hline & $\operatorname{LSD}(0.05)$ & \multicolumn{4}{|c|}{1.641} \\
\hline \multirow[t]{4}{*}{$2013-14$} & $\mathrm{M}_{1}$ (No manure) & 6.16 & 11.76 & 17.29 & 21.92 \\
\hline & M & 7.02 & 12.27 & 24.86 & 44.94 \\
\hline & SEm \pm & \multicolumn{4}{|c|}{1.032} \\
\hline & LSD (0.05) & \multicolumn{4}{|c|}{3.012} \\
\hline \multirow[t]{4}{*}{ 2014-15 } & $\mathrm{M}_{1}$ (No manure) & 4.94 & 6.35 & 17.21 & 17.76 \\
\hline & M2 (FYM @5t/ha) & 6.16 & 8.00 & 29.45 & 17.25 \\
\hline & SEm \pm & \multicolumn{4}{|c|}{0.748} \\
\hline & LSD $(0.05)$ & \multicolumn{4}{|c|}{2.185} \\
\hline \multirow[t]{3}{*}{$2015-16$} & $\mathrm{M}_{1}($ No manure $)$ & 3.37 & 4.24 & 7.57 & 10.62 \\
\hline & $\mathrm{SEm} \pm$ & \multicolumn{4}{|c|}{0.713} \\
\hline & LSD (0.05) & \multicolumn{4}{|c|}{2.081} \\
\hline \multirow[t]{4}{*}{ 2016-17 } & $\mathrm{M}_{1}$ (No manure) & 3.37 & 4.23 & 6.24 & 8.62 \\
\hline & M2 (FYM @ 5t/ha) & 3.88 & 4.59 & 8.39 & 8.47 \\
\hline & SEm \pm & \multicolumn{4}{|c|}{0.332} \\
\hline & LSD (0.05) & \multicolumn{4}{|c|}{0.970} \\
\hline \multirow[t]{4}{*}{ 2017-18 } & $\mathrm{M}_{1}$ (No manure) & 2.00 & 2.19 & 2.39 & 2.39 \\
\hline & M2 (FYM @ 5t/ha) & 1.96 & 2.23 & 3.84 & 2.74 \\
\hline & $\mathrm{SEm} \pm$ & \multicolumn{4}{|c|}{0.119} \\
\hline & $\operatorname{LSD}(0.05)$ & \multicolumn{4}{|c|}{0.348} \\
\hline \multirow[t]{4}{*}{ Total } & $\mathrm{M}_{1}$ (No manure) & 25.45 & 36.31 & 68.00 & 81.45 \\
\hline & M2 (FYM @5t/ha) & 30.82 & 47.96 & 117.29 & 126.51 \\
\hline & SEm \pm & \multicolumn{3}{|c|}{1.294} & \\
\hline & LSD (0.05) & \multicolumn{3}{|c|}{3.777} & \\
\hline
\end{tabular}


Table.6 Interaction effect of drip-irrigation (I) and nutrients $(\mathrm{N})$ on number of suckers production/ha ('000) in sisal

\begin{tabular}{|c|c|c|c|c|c|}
\hline \multirow[t]{2}{*}{ Year } & \multirow[t]{2}{*}{ Treatments } & \multicolumn{4}{|c|}{ Number of suckers produced/ha ('000) } \\
\hline & & $\mathrm{N}_{0} \mathrm{P}_{0} \mathrm{~K}_{0}$ & $\mathrm{~N}_{0} \mathrm{P}_{30} \mathrm{~K}_{60}$ & $\mathrm{~N}_{60} \mathrm{P}_{30} \mathrm{~K}_{60}$ & $\mathrm{~N}_{120} \mathrm{P}_{30} \mathrm{~K}_{60}$ \\
\hline \multirow[t]{4}{*}{$2012-13$} & $\mathrm{I}_{1}$ (No irrigation) & 5.49 & 8.98 & 23.53 & 24.20 \\
\hline & $\mathrm{I}_{2}$ (Drip irrigation) & 7.18 & 12.31 & 28.66 & 31.92 \\
\hline & SEm \pm & \multicolumn{4}{|c|}{0.562} \\
\hline & LSD (0.05) & \multicolumn{4}{|c|}{1.641} \\
\hline \multirow[t]{4}{*}{ 2013-14 } & $\mathrm{I}_{1}$ (No irrigation) & 6.31 & 11.21 & 17.92 & 27.53 \\
\hline & $\mathrm{I}_{2}$ (Drip irrigation) & 6.86 & 12.82 & 24.23 & 39.33 \\
\hline & SEm \pm & \multicolumn{4}{|c|}{1.032} \\
\hline & LSD (0.05) & \multicolumn{4}{|c|}{3.012} \\
\hline \multirow[t]{4}{*}{ 2014-15 } & $\mathrm{I}_{1}$ (No irrigation) & 5.33 & 7.02 & 18.43 & 14.59 \\
\hline & $\mathrm{I}_{2}$ (Drip irrigation) & 5.76 & 7.33 & 28.24 & 20.43 \\
\hline & SEm \pm & \multicolumn{4}{|c|}{0.748} \\
\hline & $\operatorname{LSD}(0.05)$ & \multicolumn{4}{|c|}{2.185} \\
\hline \multirow[t]{4}{*}{$2015-16$} & $\mathrm{I}_{1}$ (No irrigation) & 3.65 & 4.47 & 11.45 & 11.21 \\
\hline & $\mathrm{I}_{2}$ (Drip irrigation) & 4.47 & 6.86 & 11.96 & 16.51 \\
\hline & SEm \pm & \multicolumn{4}{|c|}{0.713} \\
\hline & LSD (0.05) & \multicolumn{4}{|c|}{2.081} \\
\hline \multirow[t]{4}{*}{ 2016-17 } & $\mathrm{I}_{1}$ (No irrigation) & 3.57 & 4.00 & 5.26 & 5.76 \\
\hline & $\mathrm{I}_{2}$ (Drip irrigation) & 3.68 & 4.82 & 9.37 & 11.33 \\
\hline & SEm \pm & \multicolumn{4}{|c|}{0.332} \\
\hline & LSD (0.05) & \multicolumn{4}{|c|}{0.970} \\
\hline \multirow[t]{4}{*}{ 2017-18 } & $\mathrm{I}_{1}$ (No irrigation) & 1.96 & 2.20 & 2.70 & 2.47 \\
\hline & $\mathrm{I}_{2}$ (Drip irrigation) & 2.00 & 2.23 & 3.53 & 2.66 \\
\hline & SEm \pm & \multicolumn{4}{|c|}{0.119} \\
\hline & LSD (0.05) & \multicolumn{4}{|c|}{0.348} \\
\hline \multirow[t]{4}{*}{ Total } & $\mathrm{I}_{1}$ (No irrigation) & 26.31 & 37.88 & 79.29 & 85.76 \\
\hline & $\mathrm{I}_{2}$ (Drip irrigation) & 29.96 & 46.39 & 106.00 & 122.19 \\
\hline & SEm \pm & \multicolumn{4}{|c|}{1.294} \\
\hline & LSD (0.05) & \multicolumn{4}{|c|}{3.777} \\
\hline
\end{tabular}


Table.7 Interaction effects of manure (M), irrigation (I) and nutrients (N) on number of sucker production/ha ('000) in sisal

\begin{tabular}{|c|c|c|c|c|c|c|}
\hline \multirow[t]{3}{*}{ Year } & \multirow[t]{3}{*}{ Manure } & \multirow[t]{3}{*}{ Irrigation } & \multicolumn{4}{|c|}{ Number of sucker produced/ha ('000) } \\
\hline & & & \multicolumn{4}{|c|}{ Nutrients } \\
\hline & & & $\mathrm{N}_{0} \mathrm{P}_{0} \mathrm{~K}_{0}$ & $\mathrm{~N}_{0} \mathrm{P}_{30} \mathrm{~K}_{60}$ & $\mathrm{~N}_{60} \mathrm{P}_{30} \mathrm{~K}_{60}$ & $\mathrm{~N}_{120} \mathrm{P}_{30} \mathrm{~K}_{60}$ \\
\hline \multirow{6}{*}{$\frac{m}{\frac{\pi}{3}}$} & \multirow[t]{2}{*}{$\mathrm{M}_{1}$ (No manure) } & $\mathrm{I}_{1}$ (No irrigation) & 4.32 & 5.80 & 13.65 & 18.51 \\
\hline & & $\mathrm{I}_{2}$ (Drip irrigation) & 6.90 & 9.25 & 20.94 & 21.73 \\
\hline & \multirow[t]{2}{*}{$\mathrm{M}_{2}(\mathrm{FYM} @$ 5t/ha) } & $\mathrm{I}_{1}$ (No irrigation) & 6.67 & 12.16 & 33.41 & 29.88 \\
\hline & & $\mathrm{I}_{2}$ (Drip irrigation) & 7.45 & 15.37 & 36.39 & 42.12 \\
\hline & \multicolumn{2}{|l|}{$\mathrm{SEm} \pm$} & \multicolumn{4}{|c|}{0.795} \\
\hline & \multicolumn{2}{|l|}{$\operatorname{LSD}(0.05)$} & \multicolumn{4}{|c|}{2.321} \\
\hline \multirow{6}{*}{$\frac{⿱ 亠 凶}{\frac{\pi}{7}}$} & \multirow[t]{2}{*}{$\mathrm{M}_{1}$ (No manure) } & $\mathrm{I}_{1}$ (No irrigation) & 5.80 & 10.43 & 15.29 & 17.18 \\
\hline & & $\mathrm{I}_{2}$ (Drip irrigation) & 6.51 & 13.10 & 19.29 & 26.67 \\
\hline & \multirow[t]{2}{*}{$\mathrm{M}_{2}$ (FYM @ 5t/ha) } & $\mathrm{I}_{1}$ (No irrigation) & 6.82 & 12.00 & 20.55 & 37.89 \\
\hline & & $\mathrm{I}_{2}$ (Drip irrigation) & 7.21 & 12.55 & 29.18 & 52.00 \\
\hline & \multicolumn{2}{|l|}{$\mathrm{SEm} \pm$} & \multicolumn{4}{|c|}{1.459} \\
\hline & \multicolumn{2}{|l|}{$\operatorname{LSD}(0.05)$} & \multicolumn{4}{|c|}{ NS } \\
\hline \multirow{6}{*}{$\frac{\frac{n}{7}}{\frac{1}{8}}$} & \multirow[t]{2}{*}{$\mathrm{M}_{1}$ (No manure) } & $\mathrm{I}_{1}$ (No irrigation) & 4.71 & 6.27 & 9.88 & 9.18 \\
\hline & & $\mathrm{I}_{2}$ (Drip irrigation) & 5.18 & 6.43 & 24.55 & 26.35 \\
\hline & \multirow[t]{2}{*}{$\mathrm{M}_{2}(\mathrm{FYM} @ 5 \mathrm{t} / \mathrm{ha})$} & $\mathrm{I}_{1}$ (No irrigation) & 5.96 & 7.76 & 26.98 & 20.00 \\
\hline & & $\mathrm{I}_{2}$ (Drip irrigation) & 6.35 & 8.24 & 31.92 & 14.51 \\
\hline & \multicolumn{2}{|l|}{$\mathrm{SEm} \pm$} & & & 059 & \\
\hline & $\operatorname{LSD}(0.05)$ & & & & 090 & \\
\hline & $\mathrm{M}_{1}$ (No manure) & $\mathrm{I}_{1}$ (No irrigation) & 2.82 & 3.84 & 6.75 & 8.15 \\
\hline & & $\mathrm{I}_{2}$ (Drip irrigation) & 3.92 & 4.63 & 8.39 & 13.10 \\
\hline '̆ & $\mathrm{M}_{2}(\mathrm{FYM} @$ 5t/ha $)$ & $\mathrm{I}_{1}$ (No irrigation) & 4.47 & 5.10 & 16.16 & 14.27 \\
\hline 룽 & & $\mathrm{I}_{2}$ (Drip irrigation) & 5.02 & 9.10 & 15.53 & 19.92 \\
\hline & SEm \pm & & & & 008 & \\
\hline & $\operatorname{LSD}(0.05)$ & & & & NS & \\
\hline & $\mathrm{M}_{1}$ (No manure) & $\mathrm{I}_{1}$ (No irrigation) & 3.29 & 3.76 & 4.63 & 5.72 \\
\hline & & $\mathrm{I}_{2}$ (Drip irrigation) & 3.45 & 4.71 & 7.84 & 11.53 \\
\hline$\vec{b}$ & $\mathrm{M}_{2}(\mathrm{FYM} @$ 5t/ha $)$ & $\mathrm{I}_{1}$ (No irrigation) & 3.84 & 4.24 & 5.88 & 5.81 \\
\hline 8 & & $\mathrm{I}_{2}$ (Drip irrigation) & 3.92 & 4.94 & 10.90 & 11.14 \\
\hline & SEm \pm & & & & 469 & \\
\hline & $\operatorname{LSD}(0.05)$ & & & & NS & \\
\hline & $\mathrm{M}_{1}$ (No manure) & $\mathrm{I}_{1}$ (No irrigation) & 1.96 & 2.20 & 2.27 & 2.35 \\
\hline & & $\mathrm{I}_{2}$ (Drip irrigation) & 2.04 & 2.19 & 2.51 & 2.43 \\
\hline$\frac{\pi}{1}$ & $\mathrm{M}_{2}(\mathrm{FYM} @ 5 \mathrm{t} / \mathrm{ha})$ & $\mathrm{I}_{1}$ (No irrigation) & 1.96 & 2.20 & 3.14 & 2.59 \\
\hline 롱 & & $\mathrm{I}_{2}$ (Drip irrigation) & 1.96 & 2.27 & 4.55 & 2.90 \\
\hline & SEm \pm & & & & 173 & \\
\hline & LSD $(0.05)$ & & & & NS & \\
\hline Total & $\mathrm{M}_{1}$ (No manure) & $\mathrm{I}_{1}$ (No irrigation) & 22.90 & 32.32 & 52.47 & 61.10 \\
\hline & & $\mathrm{I}_{2}$ (Drip irrigation) & 28.00 & 40.31 & 83.53 & 101.80 \\
\hline & $\mathrm{M}_{2}(\mathrm{FYM} @$ @ t/ha) & $\mathrm{I}_{1}$ (No irrigation) & 29.73 & 43.45 & 106.12 & 110.43 \\
\hline & & $\mathrm{I}_{2}$ (Drip irrigation) & 31.92 & 52.47 & 128.47 & 142.59 \\
\hline & SEm \pm & & & & 829 & \\
\hline & $\operatorname{LSD}(0.05)$ & & & & $\mathrm{NS}$ & \\
\hline
\end{tabular}


Interaction effect of drip-irrigation and nutrients on numbers of sucker production

Interaction of drip-irrigation and nutrients exerted significant positive effect on number of sucker production in sisal (Table 6). In 2012-13, the highest number of suckers was produced with $\mathrm{N}_{120} \mathrm{P}_{30} \mathrm{~K}_{60}$ and drip-irrigation $\left(31.92 \times 10^{3}\right)$. In the subsequent years also $\mathrm{N}_{120} \mathrm{P}_{30} \mathrm{~K}_{60}$ (and $\mathrm{N}_{60} \mathrm{P}_{30} \mathrm{~K}_{60}$ in 2017-18) nutrients along with drip irrigation produced the maximum number of suckers in sisal. For the 6 years period, a total of $122.19 \times 10^{3}$ suckers were produced from $\mathrm{N}_{120} \mathrm{P}_{30} \mathrm{~K}_{60}$ nutrients along with drip irrigation.

Interaction effect of manure, dripirrigation and nutrients on numbers of sucker production

Interaction effect of manure, irrigation and nutrients on number of sucker production in sisal were significant in the first (2012-13) and third year (2014-15) of sucker collection; and in other years, the interaction effects of the 3 factors (manure $\mathrm{x}$ irrigation $\mathrm{x}$ nutrients) were not significant on the number of sucker production (Table 7). In 2012-13, manuring with drip irrigation and the highest nutrient dose $\left(\mathrm{N}_{120} \mathrm{P}_{30} \mathrm{~K}_{60}\right)$ produced the maximum number of suckers $\left(42.12 \times 10^{3}\right)$ which was $15.7 \%$ more as compared to next lower nutrient dose $\left(\mathrm{N}_{60} \mathrm{P}_{30} \mathrm{~K}_{60}\right)$. Over the 6 years period, the maximum total sucker production $\left(142.6 \times 10^{3}\right)$ was obtained from $\mathrm{N}_{120} \mathrm{P}_{30} \mathrm{~K}_{60}$ nutrient along with manuring (FYM @ $5 \mathrm{t} / \mathrm{ha}$ ) and drip irrigation.

To conclude it may be mentioned that application of manure, drip irrigation and nutrients singly or in combination had significant positive effect on number of sucker production in sisal. For a 6 years period, combined application of manure (FYM @ $5 \mathrm{t} / \mathrm{ha}$ ), irrigation (discharge rate of $4 \mathrm{l} / \mathrm{hr}$ for 2 hours at 2 weeks interval during $1^{\text {st }}$ April to $15^{\text {th }}$ June $)$ and nutrients $\left(\mathrm{N}_{60} \mathrm{P}_{30}\right.$ $\mathrm{K}_{60}$ or $\mathrm{N}_{120} \mathrm{P}_{30} \mathrm{~K}_{60}$ ) could produce $142.6 \times 10^{3}$ suckers, which is at least 5 times more as compared to the sucker production with no manuring, no irrigation and no fertilizer application $\left(22.9 \times 10^{3}\right)$. It may be concluded that for obtaining the highest number of suckers in sisal, application of manure (FYM @ $5 \mathrm{t} / \mathrm{ha}$ ), drip irrigation (discharge rate of 4 $1 / \mathrm{hr}$ for 2 hours at 2 weeks interval during $1^{\text {st }}$ April to $15^{\text {th }}$ June) along with nutrients @ $\mathrm{N}_{60}$ $\mathrm{P}_{30} \mathrm{~K}_{60}$ or $\mathrm{N}_{120} \mathrm{P}_{30} \mathrm{~K}_{60}$ are needed. So, for 6 years, the total sucker production will be $142.6 \times 10^{3}$ per ha which is sufficient to cover about28.5 ha (@5000 suckers/ha) of new sisal plantation. Moreover, the produced suckers could add sisal planters/ farmer's income by about ₹ 5.7 lakh (@ ₹ 4 per piece of sucker) when it sold as planting material to other sisal farmers/ planters.

\section{Acknowledgement}

The authors express sincere thanks to the Director, ICAR-CRIJAF, Barrackpore, Kolkata for permitting to conduct the study and also thankful to Dr. D.K. Kundu, HQ Incharge of Sisal Research Station for providing all physical facilities required for the field experiment. Thanks are also due to Mr. M.K Pradhan, Technical Officer of Sisal Research Station, Bamra, Sambalpur, Odisha for his untiring efforts to manage the experiment and meticulous collection of field data.

\section{References}

Reddy, K.K. 1966. Cultivation of Agave sisalana in the dry tracts of Andhra Pradesh. Indian Forester, 92 (9): 596603.

Sarkar, S. 2015. Sisal: Its scope as a multidimensional fibre crop for India. Indian Farming, 65 (5): 2-7. 
Sarkar, S. and Jha, A.K. 2017. Research for sisal (Agave sp.) fibre production in India. International Journal of Current Research, 9 (11): 61136-61146.

Sarkar, S., Kar, C.S., Sinha, M.K. and Mahapatra, B.S. 2010. Improved production technology of sisal. Indian Farming, 59 (12): 17-21.
Sarkar, S., Saha, A.R. and Majumdar, B. 2017. Sucker production potential of sisal (Agave sisalana Perr. Ex Engelm.) as influenced by drip-irrigation, manure and fertilizers. International Journal of Current Agricultural Research, 5 (6): 221-223.

\section{How to cite this article:}

Sitangshu Sarkar, A.K. Jha, Bijan Majumdar and Saha, A.R. 2018. Influence of Drip-irrigation, Manure and Fertilizers on Production of Planting Material in Sisal (Agave sisalana Perr. Ex Engelm.). Int.J.Curr.Microbiol.App.Sci. 7(09): 1934-1941.

doi: https://doi.org/10.20546/ijcmas.2018.709.235 